\title{
Implementation and Validation of the Generic WECC Photovoltaics and Wind Turbine Generator Models in Modelica
}

\author{
Maria Nuschke $^{1}$ Sören Lohr ${ }^{1} \quad$ Adrien Guironnet $^{2} \quad$ Marianne Saugier $^{2}$ \\ ${ }^{1}$ Fraunhofer Institute for Energy Economics and Energy System Technology IEE, Power System Stability and \\ Converter Technology Division, Germany, \{maria.nuschke, soeren.lohr\} @iee.fraunhofer. de \\ ${ }^{2}$ Réseau de Transport d'Electricité, France, \{adrien.guironnet, marianne.saugier\}@erte-france.com
}

\begin{abstract}
This paper presents the open-source implementation in Modelica of the generic photovoltaics and wind turbine generator models introduced by the Western Electricity Coordinating Counsil (WECC) Renewable Energy Modeling Task Force. These dynamic models have been designed to be easily understandable and reusable by adopting the same decomposition as in the original WECC reports. It uses as much as possible existing Modelica Standard Library blocks and extends common parts whenever possible. The simulation results obtained with OpenModelica ${ }^{1}$ and Dyna $\omega \mathrm{o}^{2}$ - an hybrid C++/Modelica open source suite of simulation tools for power systems - have been successfully validated against different reference tools.

Keywords: Power System Modeling, Renewable Energy Sources, PV Models, Wind Turbine Generator Models, Open-Source
\end{abstract}

\section{Introduction}

Power system stability is challenged by increasing shares of Inverter-Based Generation (IBG) and systems operators' access to models representing the dynamic behavior of IBG realistically is fundamental in order to ensure a secure and safe network operation. On the other side, the exact implementation of the actual plant and generator control is treated as confidential by inverter manufacturers. Therefore, several efforts have been conducted in the past few years to propose generic or standard models for IBG. The large-scale Photovoltaics (PV) and Wind Turbine Generator (WTG) models proposed by the Western Electricity Coordinating Council (WECC) Renewable Energy Modeling Task Force (REMTF) (Ellis and et.al. 2012) are modular open-source models that enable users to perform stability studies while considering the dynamic behavior of most large PV farms and WTG installations realistically and independently from specific vendors.

Since the first definition of the generic WECC PV and WTG models in 2012, several implementations in commercial software environments have been presented, e.g. (Gustav Lammert, Luis David Pabon Ospina, et al. 2016)

\footnotetext{
${ }^{1}$ https: / / www. openmodelica.org

${ }^{2}$ http: //www.dynawo.org
}

and various stability studies have been demonstrating the dynamic behaviour of these models under different conditions realistically, e.g. (G. Lammert, L. D. Pabon Ospina, and al. 2017; Gustav Lammert, Premm, et al. 2017; Luis David Pabon Ospina et al. 2018; Nuschke et al. 2019; L. D. Pabon Ospina and T. V. Cutsem 2020; L. D. Pabon Ospina and T. Cutsem 2020).

Further improvements to the models have also been applied. For instance, a new voltage sourced interface (Pourbeik 2018; Ramasubramanian et al. 2017) as an extension to the conventionally used current source interface has been proposed to improve the numerical stability of the simulation in situations with very high shares of IBG.

In the meantime, Modelica has gained a growing interest in the power system community. In addition to already existing efforts driven by first-hours Modelica enthusiasts in the Modelica.Electrical.QuasiStationary or PowerSystems libraries, the European projects Pegase and iTesla have boosted the use of Modelica in the power system community. They notably contribute to prove the language usability for power system modeling (Chieh, Panciatici, and J.Picard 2011) and affirm its interest for unambiguous models implementation (Vanfretti et al. 2013). Since these projects end, more and more power system stakeholders are using Modelica either for academic works (Gonzalez-Torres et al. 2019; Mirz et al. 2019; Qin et al. 2019; Masoom et al. 2020) or industrial use (Casella et al. 2016; Guironnet, Saugier, et al. 2018; Guironnet, Rosière, and Bureau 2021). They are attracted by the flexibility, usability and robustness of the language coupled with the progresses done in Modelica tools for largescale simulations (Braun, Casella, and Bachmann 2017; Henningsson, Olsson, and Vanfretti 2019), and the creation and availability of generic and easy to adopt libraries such as the PowerGrids (Bartolini, Casella, and Guironnet 2019) or Dyna $\omega$ o (Guironnet, Saugier, et al. 2018) ones.

One key aspect favorizing the spread of the Modelica language in the power system community is undoubtedly its easiness for the modeling of non conventional components and usefulness for stability and design studies of such components. Indeed, the flexibility and freedom offered in the models development - no constraint on the interfaces, possibility to mix block and equation-based approaches, etc. - but also in the test case creation - possi- 


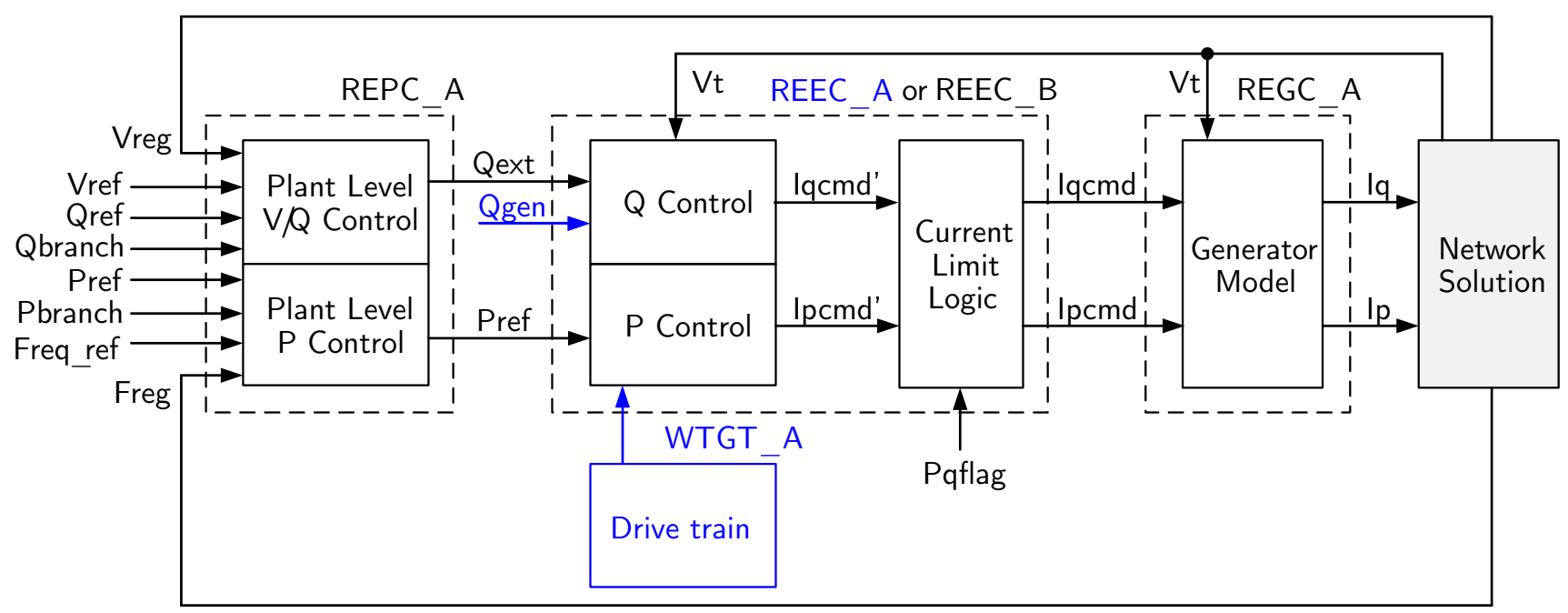

Figure 1. WECC block diagram PV (black), with additional blocks for WTG (blue), see Ellis and et.al. (2012)

bility to mix different kind of models for example - make it a good candidate for advanced studies. It is in particular the case in the field of power-electronics dominated systems as shown in (Cossart, Rosiere, et al. 2020). The release of different open-source components, e.g. (Murad, Gomez, and Vanfretti 2015; Cossart, Saugier, and Guironnet 2021), and use cases is one additional point to promote the use of the Modelica language in this direction.

The contribution of this paper is thus to present an implementation of the generic large-scale WECC PV and WTG models in Modelica and their validation against reference tools. To the best of the authors' knowdlege, it is the first open-source implementation of these models. Furthermore, their integration into the Dynawo suite - the models are available in the Modelica library of the project 3 - enables to offer a wide variety of test cases in which these models can be integrated.

The rest of this paper is organized in the following way. Section 2 is devoted to the WECC PV and WTG models presentation while Section 3 describes their implementation. The design choices, the modeling approach and the implementation of a few selected components are detailed in this later section. Section 4 demonstrates the excellent level of accuracy obtained with the models by a comparison with reference results from a validation tool provided by EPRI as well as from the standard library object provided in DIgSILENT/PowerFactory. Finally Section 5 gives the conclusion.

\section{Models Presentation}

The WECC models and their updates are described in specific modeling guidelines and publications, e.g. (Ellis and et.al. 2012; Pourbeik 2018; Ramasubramanian et al. 2017). For convenience, the description of the most relevant blocks is given in the sequel.

\footnotetext{
${ }^{3}$ https://github.com/dynawo/dynawo/tree/ master/dynawo/sources/Models/Modelica/Dynawo
}

\subsection{Overview}

The WECC PV and WTG type 4 (Type 4 is considered as fully rated converter WTG) models share a common highlevel organization, as depicted in Figure 1 and are divided in three main control blocks:

- The plant control - called Renewable Energy Plant Control (REPC) - sets the main control choice for the whole plant. Voltage or reactive power control at plant level and frequency-dependent active power adjustment are part of the plant level. Identical in PV and WTG models.

- The electrical control - called Renewable Energy Electrical Control (REEC) - includes local inverter functionalities such as Fault-Ride Through (FRT) characteristic with fast reactive current injection, local voltage and reactive power control and current limitation with respect to the priority given to active or reactive current, respectively. There are two module versions available, whereas the REEC_B module is recommended for the WECC PV models and the REEC_A module is recommended for WECC WTG. The REEC_B module is a slightly simplified version from REEC_A module.

- The generator control - called Renewable Energy Generator Control (REGC) - is the last part of the control and interfaces with the grid. It enables to convert the current set-points calculated by the REEC part into the final currents (or voltages) delivered to the network. Identical in PV and WTG models.

For the WTG model a drive-train model - called Wind Turbine Generator Train (WTGT) - can be considered additionally in order to represent rotor speed changes and possibly resulting torsional oscillations after faults or sudden wind speed changes. By considering the drive-train model, the WTG model is equivalent to the WECC WTG 
type 4A. By neglecting the drive-drain model, the rotor speed is considered to be constant at nominal value 1 p.u. and the WTG model is equivalent to the WECC WTG type 4B. The model types $4 \mathrm{~A}$ and $4 \mathrm{~B}$ are sub-types of the fully rated converter model for wind turbine generators.

\subsection{Plant level control (REPC)}

The plant level control determines the local inverter setpoints for active power (Pref) and reactive power (Qext) as input to the REEC by considering measurements at Point of Common Coupling (PCC) and user-defined setpoints at plant level.

The active power regulation is shown in Figure 2. Frequency dependent active power adjustment can be activated by setting the FrqFlag to true and is done by a proportional integer action on an addition of the frequency deviation and active power injection deviation. Note that if the adjustment is deactivated, Pref will be directly passed to the REEC.

The reactive power regulation control is displayed in Figure 3. Reactive power control can be realized with reference either to reactive power or to voltage amplitude but is always done with a proportional integer action. The choice is made with the parameter RefFlag. Typically, in voltage control mode, the regulated bus is the point of common coupling and measurement values are available from that bus. The regulated bus can also be chosen different from the PCC. Therefore, the parameter V cmpFlag has to be set to true and the impedance $R c+j X c$ specifies the impedance between the PCC and the desired remotely controlled bus.

\subsection{Electrical control (REEC)}

The electrical control is itself divided in two main functions:

- The first part determines from the input set-points Pref and Qext the necessary currents to inject in the network.

- The second part is the current limiter logic that will potentially limit the current injections through a certain process, that differs between the PV and WTG models.

In the $\mathrm{P}$ control, the currents direct component ipcmd is calculated through a first-order structure with limits on $\mathrm{P}$ and its derivative and then on the current value itself. Note that the variation can be frozen by an external signal, in case of a voltage dip.

In the $\mathrm{Q}$ control, the overall structure is more complex and handles different kind of control modes. If a local coordinated V/Q control is activated on top of the plant control, there are different local proportional-integral actions applied on the input signal. Otherwise, the control is similar to the $\mathrm{P}$ section with a first-order structure. Both control loops (with or without local coordinated V/Q control) can be frozen by an external loop signal, in case of a voltage dip. Finally, the last part of the control structure the upper part - corresponds to an additional current injection that is activated in FRT situations. All these loops are visible in Figure 4.

Regarding the current limiter logic, the priority between active and reactive support is defined through a flag. If the flag prioritizes the active current injection, the limits are defined in the following way:

$$
\begin{aligned}
\text { ipmax } & =\text { imax } \\
\text { ipmin } & =0 \\
\text { iqmax } & =\sqrt{\left(\text { imax }^{2}-i p c m d^{2}\right)} \\
\text { iqmin } & =- \text { iqmax }
\end{aligned}
$$

Vice versa, in case of priority given to reactive current injection, the active current component will be reduced in favor of reactive current.

The reactive current injection during faults (Fault ride through capability, FRT) is implemented as a voltage dependent current injection for the PV model. For the WTG model, the FRT behavior is defined by three potential states, as depicted in Figure 5, where either no injection, voltage dependent injection or a constant injection is made. The parameter Thld decides what happens after a voltage dip has ended.

\subsection{Generator control (REGC)}

The generator control REGC_A as per the initial implementation proposal from WECC (Ellis and et.al. 2012) calculates the set-points for active and reactive current considering ramp rate limiters for the currents or active power. The enhancements introduced in (Pourbeik 2018) including reference voltage calculation or even current control and phase-locked-loop are represented in different sub-modules REGC_B and REGC_c, respectively.Rotor

\subsection{Control modes}

As already shown in the previous parts, the models offer several degrees of freedom and enable to activate or deactivate different kinds of controls by changing the values of the corresponding flags. Table 1 illustrates this with a few examples that allow to model different strategies for voltage or reactive power control.

\section{Modelica Implementation}

\subsection{Design choices}

In the context of the WECC models and considering the models structure presented in the previous section, the implementation in Modelica has been done in the following way:

- The injector that connects the PV or WTG model to the network, as well as the voltage drop between the PCC and the voltage set-point or the current limitations logics are described through equations as specific sub-components. 


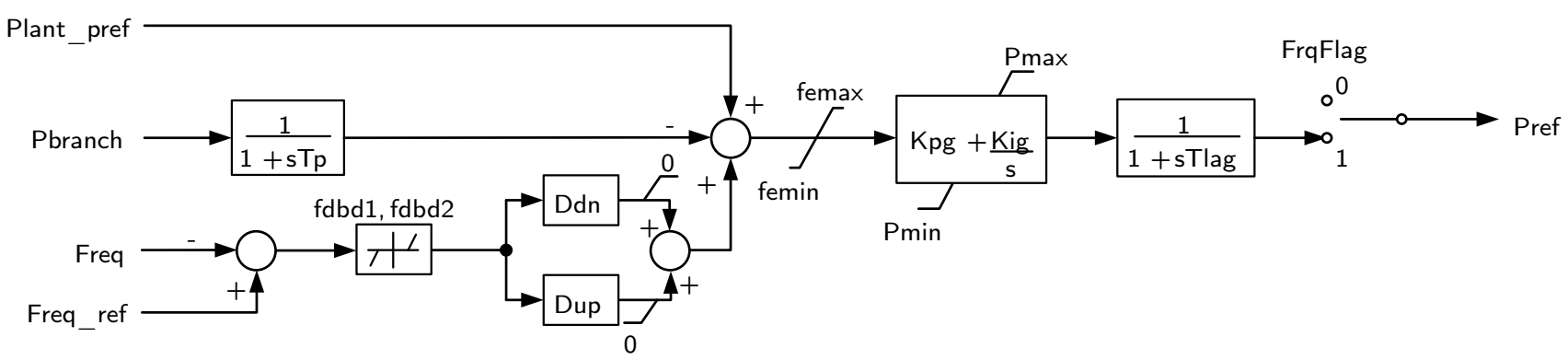

Figure 2. REPC - Active power control according to Ellis and et.al. (2012)

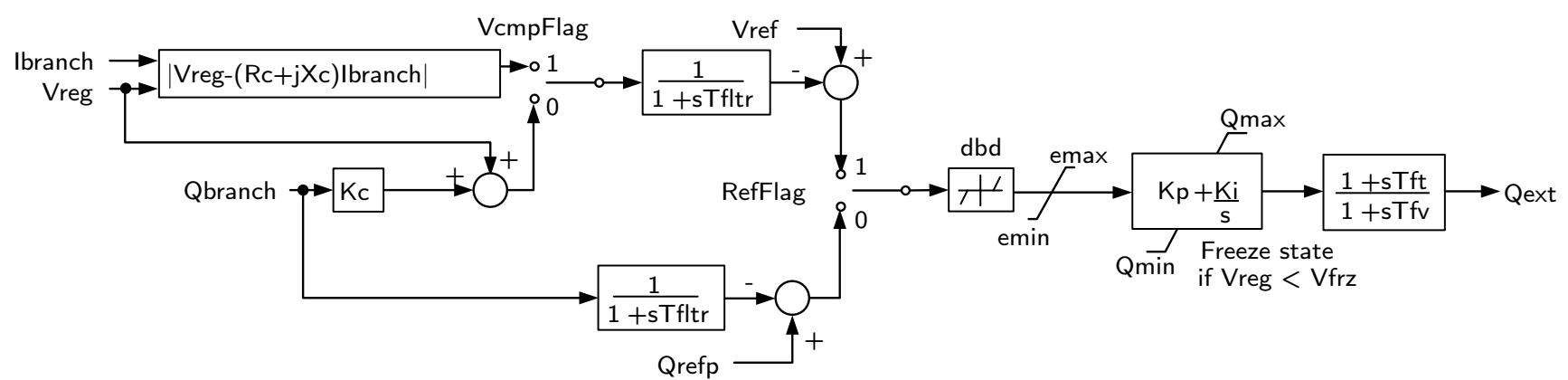

Figure 3. REPC - Reactive power control according to Ellis and et.al. (2012)

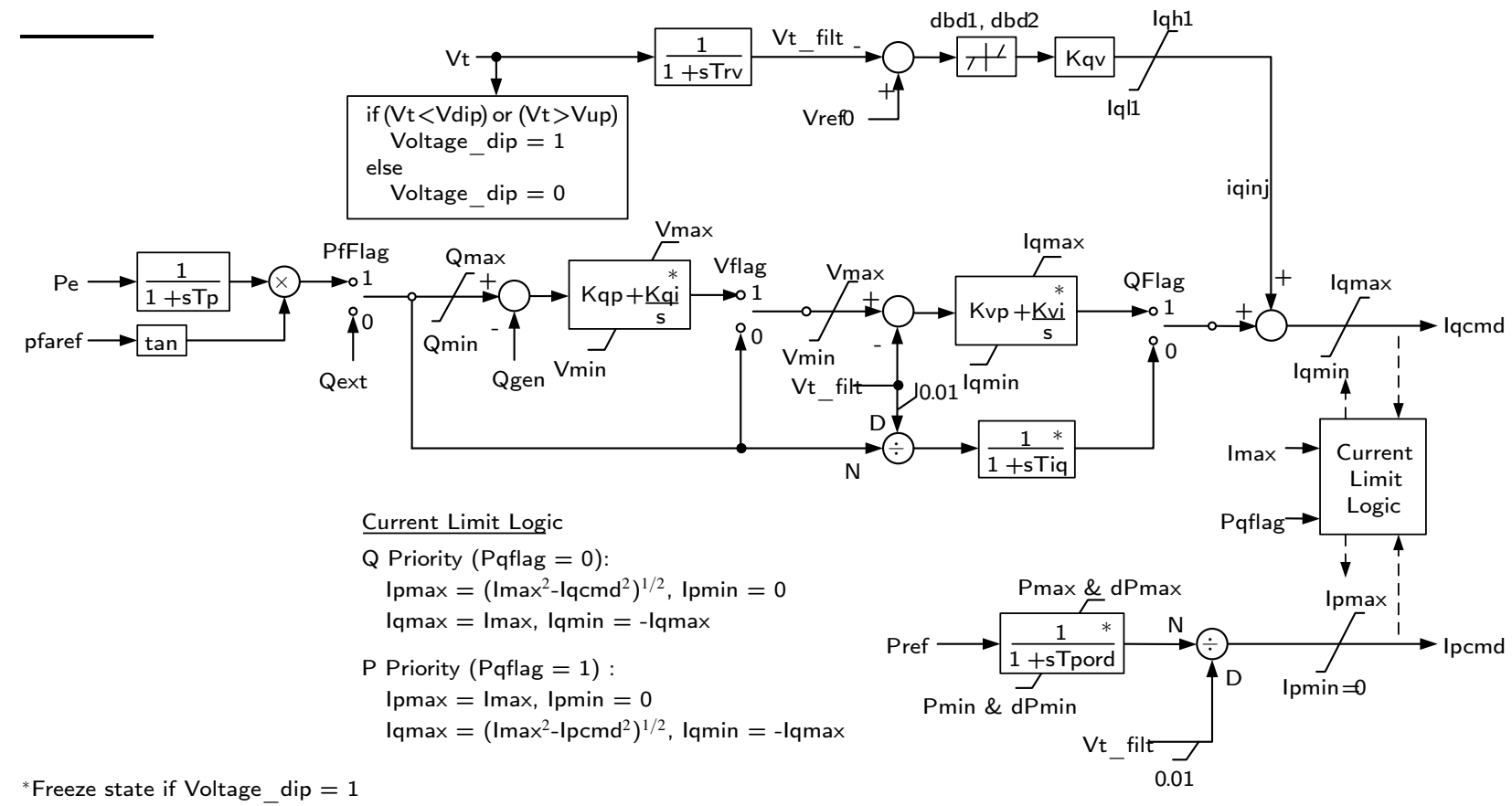

Figure 4. REEC_B - Electrical control according to Ellis and et.al. (2012)

- The other parts of the control are split in a similar way to the WECC original models, based on their behavior, and are described through a diagram approach.

The major parts of the models are thus built by combining individual and elementary blocks in a very similar way to the original WECC documentation (Ellis and et.al. 2012).
In order to ease the long-term maintenance and to benefit from the robustness of widely used components, the Modelica Standard Library has been used as much as possible in the models.

Complementary blocks have been developed to handle specificities of the WECC models, such as the possibility to freeze the block actions with an external order sig- 
Table 1. Control functionalities with plant level control and electrical control in service Ellis and et.al. (2012)

\begin{tabular}{clllll}
\hline No. & Control mode & PFFlag & VFlag & QFlag & RefFlag \\
\hline 1 & Plant level Q control & 0 & N/A & 0 & 0 \\
2 & Plant level V control & 0 & N/A & 0 & 1 \\
3 & Plant level Q control with local coordinated V/Q control & 0 & 1 & 1 & 0 \\
4 & Plant level V control with local coordinated V/Q control & 0 & 1 & 1 & 1 \\
\hline
\end{tabular}

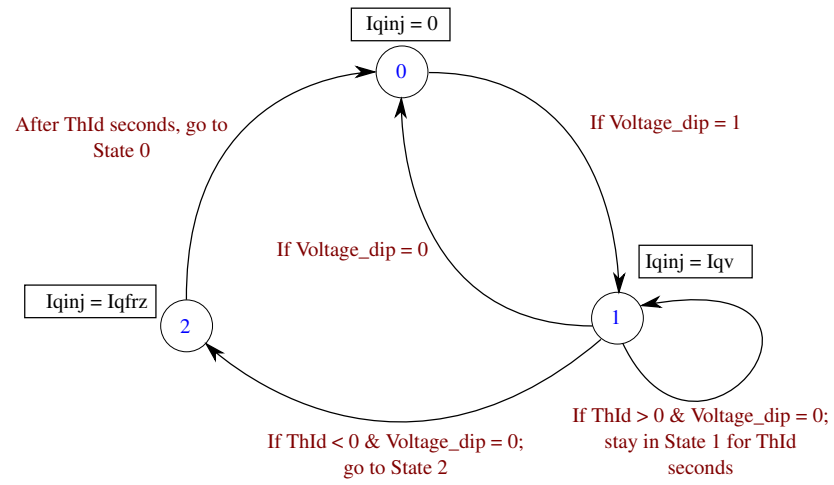

Figure 5. FRT behavior for the WECC WTG model according to Ellis and et.al. (2012)

nal. These additional blocks have been kept as generic as possible to facilitate their reuse through the whole models implementations.

\subsection{Architecture}

PV and WTG models have a very common structure, composed of identical REPC and REGC controls. Only part of the REEC control is different between the two generic models, and WTG model also has one additional structure to represent the drive-train behavior.

To minimize the number of models developed and reuse as much as possible common parts, the REPC and REGC controls have been developed only once. A common basis has also been defined for the REEC control, enabling to extend it to build the two final REEC control blocks REEC_B and REEC_A for PV and WTG models, respectively. It is worth mentioning that a large part of the block is implemented in the common basis. The same approach has been used for two possible implementations of the WTGT block.

Finally, the complete PV or WTG models are obtained by combining the different parts of the control with an injector providing the currents to the network. With such an architecture, modifying one part of the model or adding a new block is very easy and straightforward; it can be done without any modifications on the other parts.

\subsection{Illustration}

Figure 6 depicts the overall composite model, the similarity to Figure 1 is obvious. Further, detailed Modelica implementations of some WECC model blocks are given.

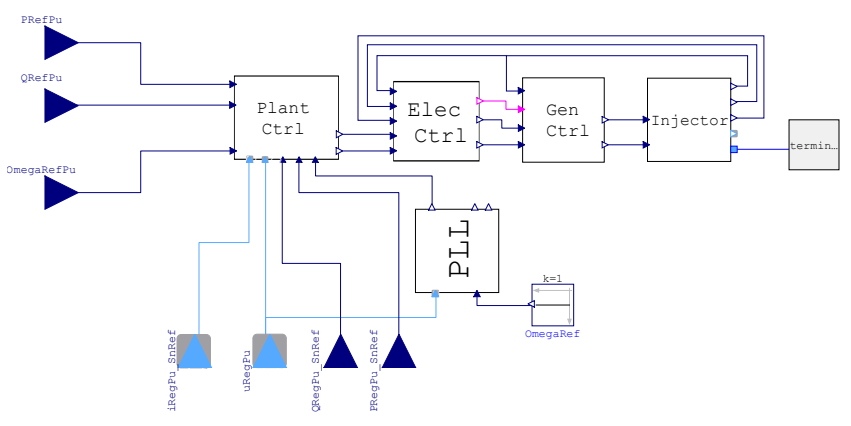

Figure 6. Block diagram of the WECC Large-scale PV model in Modelica, see Figure 1 for comparison.

\subsubsection{Plant level control (REPC)}

Figure 7 shows the implementation in Modelica of the REPC control: only the three blocks in blue have been specifically implemented for this control and are not part of the Modelica Standard Library. They represent the calculation of the voltage drop for the voltage control at a remote bus, the activation or deactivation of the freeze feature in case of voltage dip or increase and a modified implementation of a PID block to take into account the integrator state freeze.

The voltage drop calculation for voltage control at a remote busbar, is for example a short block containing the following equations:

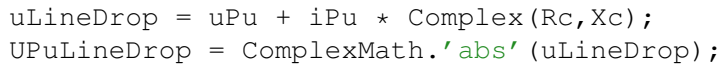

\subsubsection{Electrical control (REEC)}

Figure 8 depicts the Modelica implementation for the REEC of the WTG model. The parts surrounded in green are the ones specific for the WTG model while the others are inherited from the common control defined for both the PV and WTG models. A large part of the control is thus implemented only once in the base model.

The FRT logic shown in Figure 5 has been implemented using an algorithm approach enabling to handle the different transitions between states.

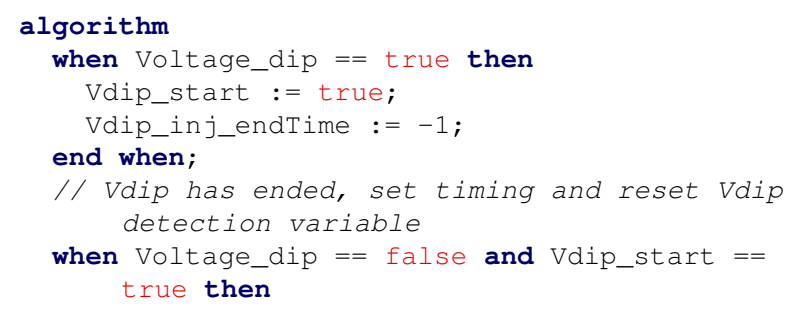




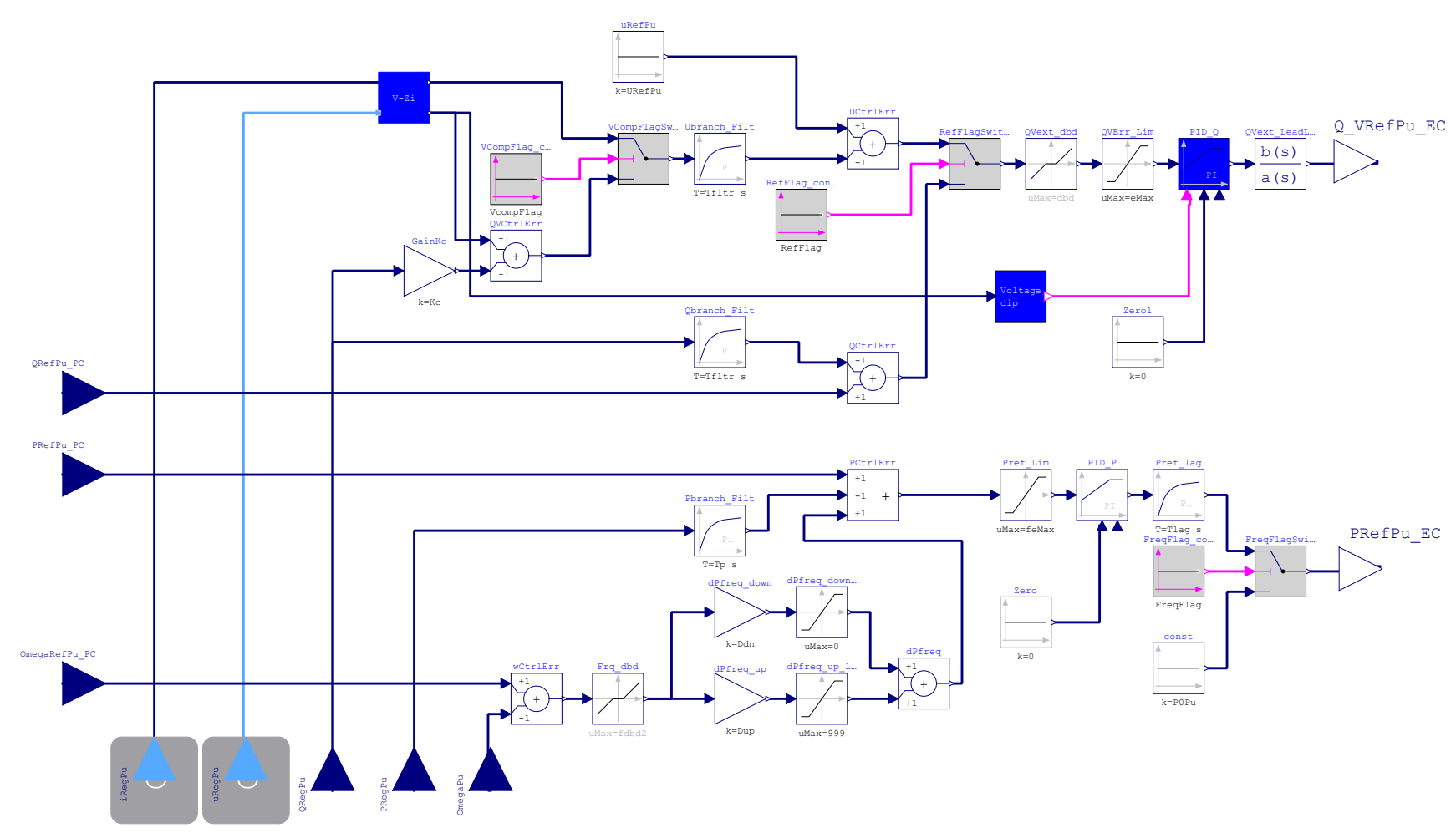

Figure 7. REPC - Park level control in Modelica, see Figures 2 and 3 for comparison.

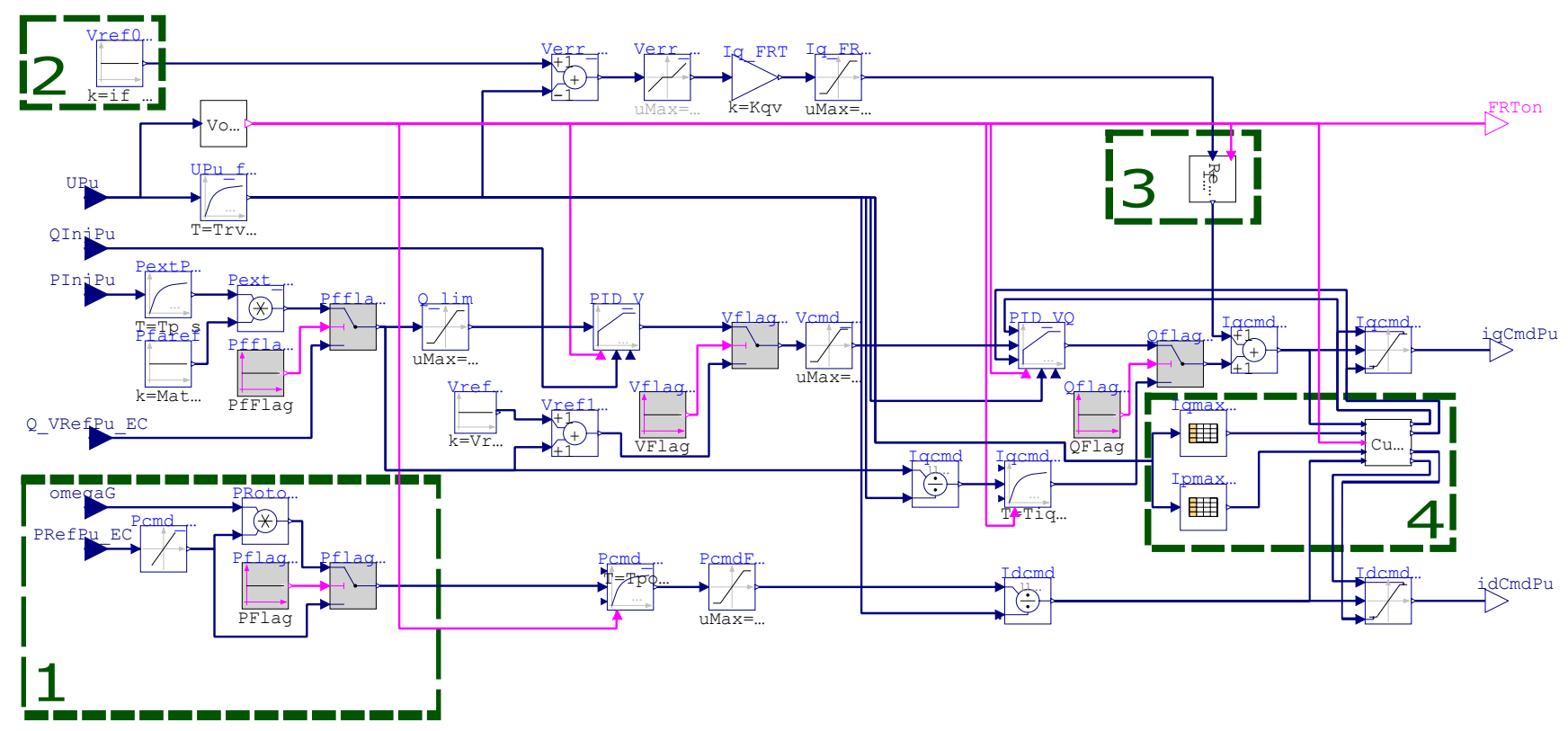

Figure 8. REEC - Electrical control in Modelica, green boxes highlight the specific blocks for the WECC WTG models: 1 consideration of variable generator speed, 2 - additional voltage reference, 3 - extended FRT logic, 4 - extended current limitation logic, see Figure 4 for comparison.

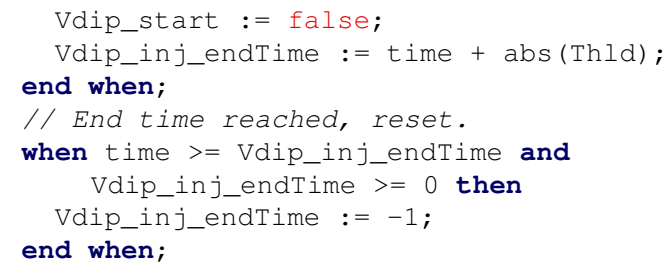

The current limit logic implementation for the WTG
REEC model, that takes into account the existence of a voltage dip or not, is presented below. It is a mix between an algorithm part to detect the voltage dip and an if clause to choose the correct minimum and maximum values depending on priority given to active or reactive power.

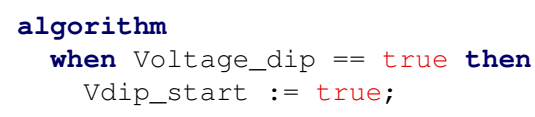




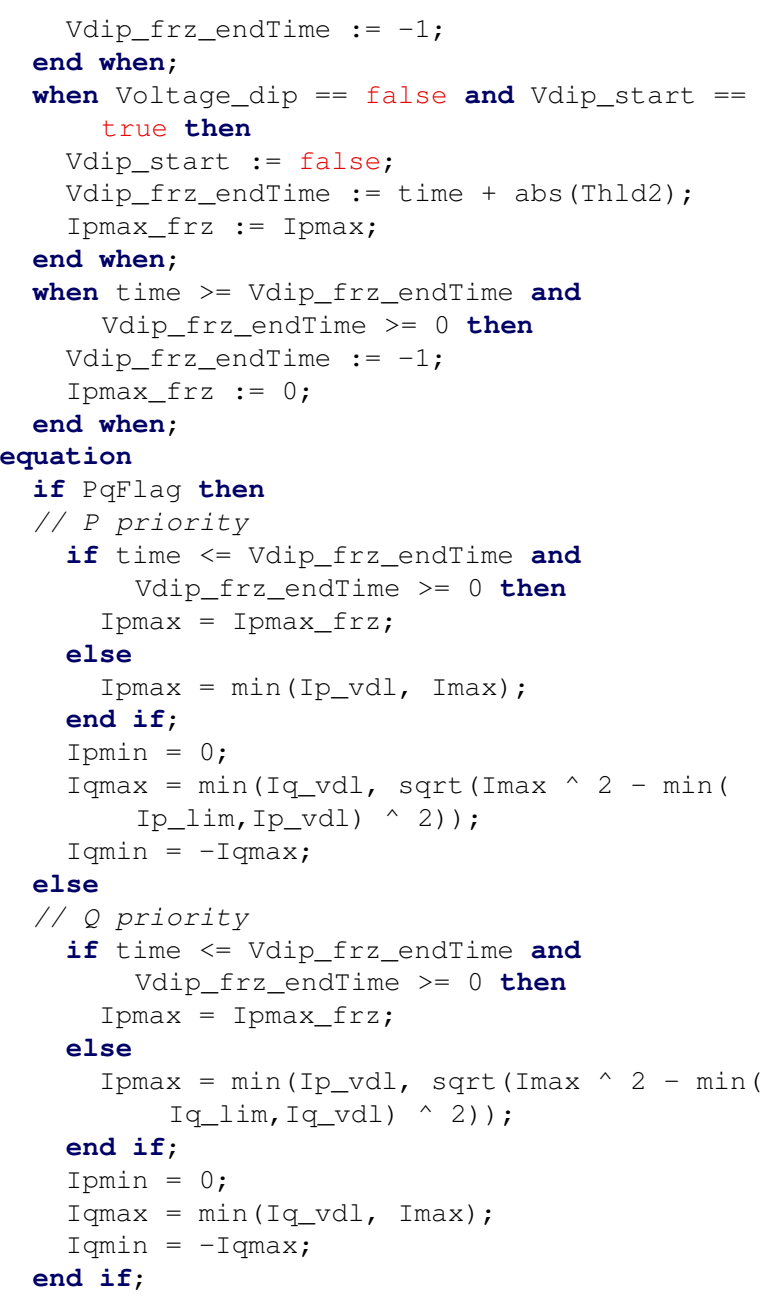

\subsubsection{Wind generator turbine drive-train (WTGT)}

The drive train model is a simplified model for the purpose of emulating the behavior of torsional mode oscillations. The shaft damping coefficient (Dshaft) in the drive-train model is fitted to capture the net damping of the torsional mode seen in the post fault electrical power response. The mechanical power $P m$ is initialized with the initial value of the electrical power $P e$. For this implementation proposal in Modelica, the block diagram approach has been chosen in order to follow the original WECC reference implementation. It is also possible to use either physical Modelica components or go for an equation-based modeling.

\subsubsection{Current injector}

In the initial model proposed by the WECC, the interface to the network is a current source, therefore a current injector element has been implemented in Modelica. It means that the REGC control calculates the d-axis and qaxis currents that are then converted to the actual currents injected to the network by a current injector using the Park transformation:

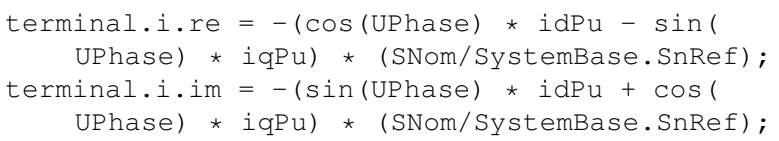

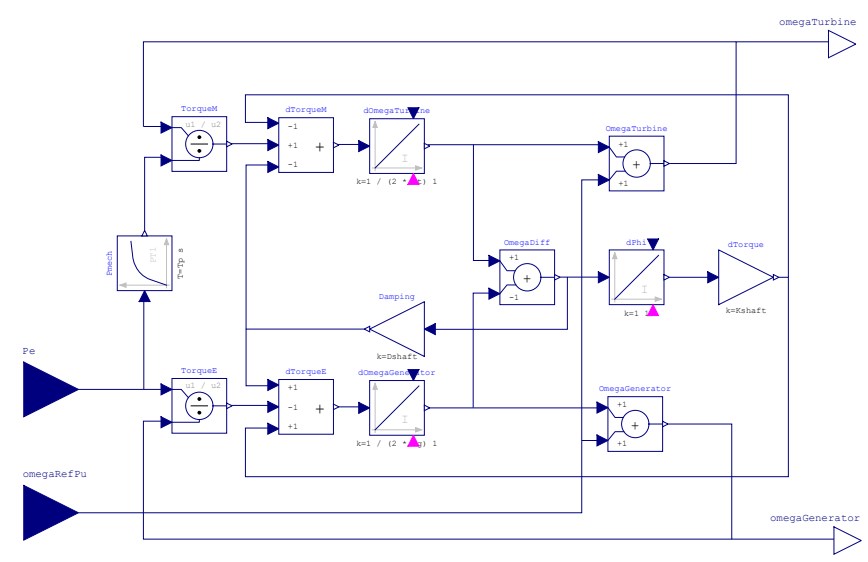

Figure 9. WTGT - Drive train model in Modelica

\subsubsection{Voltage injector}

Another interface based on directly imposing the voltage at the PCC has been introduced by the WECC, notably for higher numerical stability with very high shares of IBG. In this case, an additional block is added to convert the $\mathrm{d}$-axis and q-axis currents into inner real and imaginary voltage set-points that are then imposed to the network by a simple voltage injector, that sets the real and imaginary terminal voltages directly, see Figure 10.

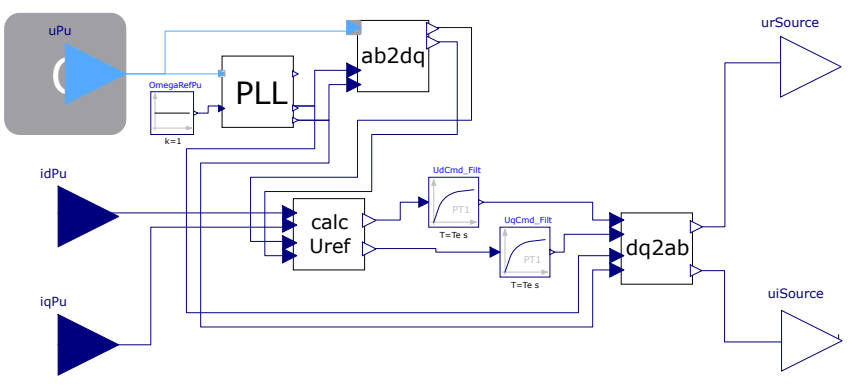

Figure 10. Voltage source reference in Modelica

The calculation of the reference voltage in the dqreference frame is implemented with the following equations using the inverter impedance $R+j X$ :

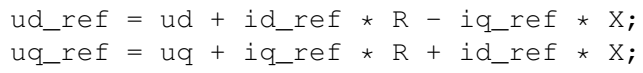

\section{Validation}

The models were validated by using the "Renewable Energy Model Validation Tool" (REMVT), written by EPRI, and additionally against a standard library implementation in DIgSILENT PowerFactory 2019, SP3. Notice that the REMVT itself was validated against real measurements.

The following sections briefly describe the test system used and then present the validation results by comparing simulation results from OpenModelica Connection Editor (OMEdit v1.13.2) with the results obtained from REMVT (Version 2.1) and the from DIgSILENT PowerFactory 2019 using the standard library object of the 
WECC PV model (DIgSILENT/PowerFactory 2019).

\subsection{Test case}

The following single line diagram shows the considered test system consisting of the IBG model, a step-up transformer, equivalent park lines and the grid-connection transformer (substation) connected to an infinite bus or external grid. The HV side of the substation (bus 4) is considered as PCC. The plant level control regulates active power, reactive power/voltage at PCC. For unambiguous voltage control at plant level, the PCC and the infinite bus must be separate buses.

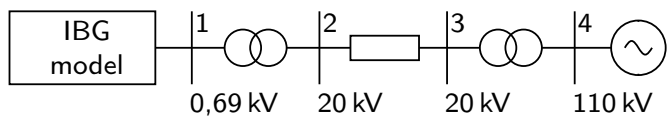

Figure 11. Single line diagram of the test system

Parameters for the equipment have been set according to Table 2. The conversion ratio for the transformers is set to one. Per unit base is SnRef $=100$ MVA.

Table 2. Test system parameters for validation

\begin{tabular}{lcr}
\hline Component & Parameter & Value in p.u. \\
\hline & $\mathrm{R}$ & 0.000100 \\
Step-up transformer, & $\mathrm{X}$ & 0.049999 \\
$20 \mathrm{kV}$ pu Base & $\mathrm{B}$ & -0.004999 \\
& $\mathrm{G}$ & 0.000110 \\
\hline & $\mathrm{R}$ & 0 \\
Line & $\mathrm{X}$ & 0.000025 \\
$20 \mathrm{kV}$ pu Base & $\mathrm{B}$ & 0 \\
& $\mathrm{G}$ & 0 \\
\hline & $\mathrm{R}$ & 0.000550 \\
Substation transformer, & $\mathrm{X}$ & 0.099999 \\
$20 \mathrm{kV}$ pu Base & $\mathrm{B}$ & -0.004999 \\
& $\mathrm{G}$ & 0.000020 \\
\hline
\end{tabular}

The test scenario consists of a voltage dip of $50 \%$ at $t=1 . .2 \mathrm{~s}$ and a frequency step up to 1.01 p.u. at $t=6 . .9 \mathrm{~s}$ at the infinite bus.

\subsection{Validation results - PV model}

This section exemplarily presents validation results from the WECC PV model with the conventional current sourced interface against the EPRI tool REMVT or the reference implementation in DIgSILENT/PowerFactory, respectively.

Figure 13 displays the validation results for control mode Nr. 1, (refering to table 1) plant level reactive power control. Simulation begins in steady state condition according to the given set-points. With the voltage dip at $t=1 . .2 \mathrm{~s}$, reactive power increases due to fast reactive current injection from FRT mode, at the same time the active
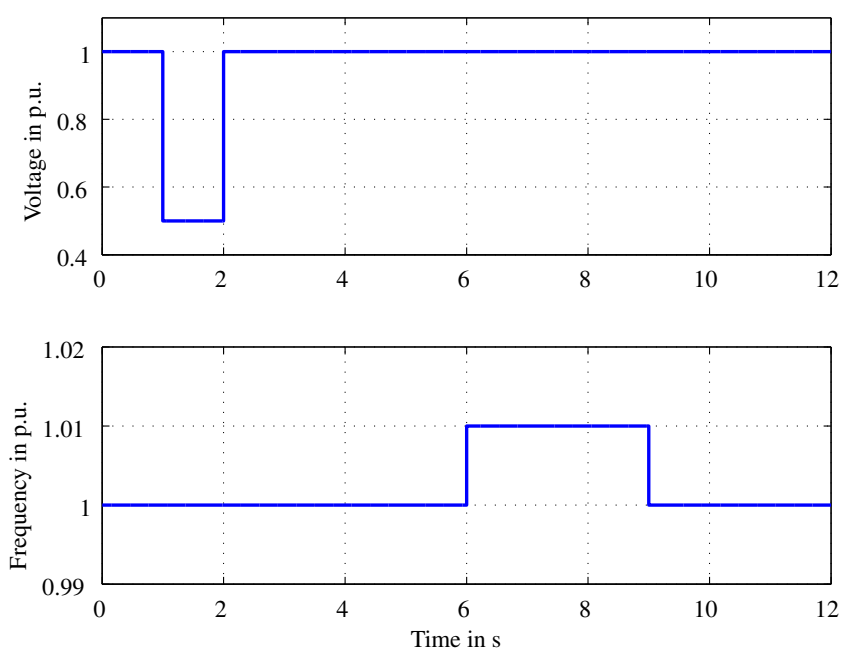

Figure 12. Test events: voltage magnitude and frequency at infinite bus terminal.

power is reduced due to the current limitation and priority given to reactive current. After voltage recovery, active and reactive power are controlled to reach the initially given set-points again.

At $t=6 \mathrm{~s}$ the active power is reduced due to the increase in frequency with respect to the given droop $D d n=20$ p.u. (pu base SNom/fNom) per frequency deviation in perunit. As the frequency at infinite bus changes back to the nominal value, active power reaches the set-point as per the initially given value.

The results from REMVT and Modelica fit very well.
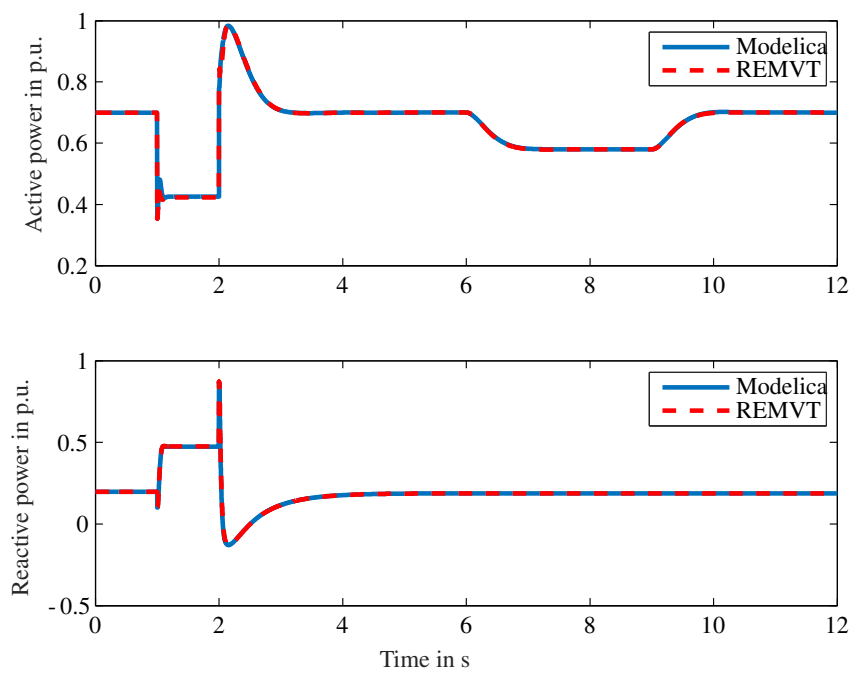

Figure 13. Simulation results $P V$ model: active and reactive power in response to test events with control mode Nr. 1

Figure 14 displays the validation results for control mode Nr. 4, plant level voltage control with local coordinated V/Q control. The active power behavior is not affected by changing the control mode from Nr. 1 to Nr. 4 and the results from REMVT and Modelica are a good fit.

Reactive power behaves similar during the voltage dip because of the still active fast reactive current injection. 
After voltage recovery, the reactive power settles slowly at a new steady state value according to the output of the plant level reactive power/voltage controller. The new steady state value is higher than the initial one because of the voltage dip: in order to support voltage, reactive power injection was increased by the plant level controller. Results from Modelica and REMVT are very well matching.
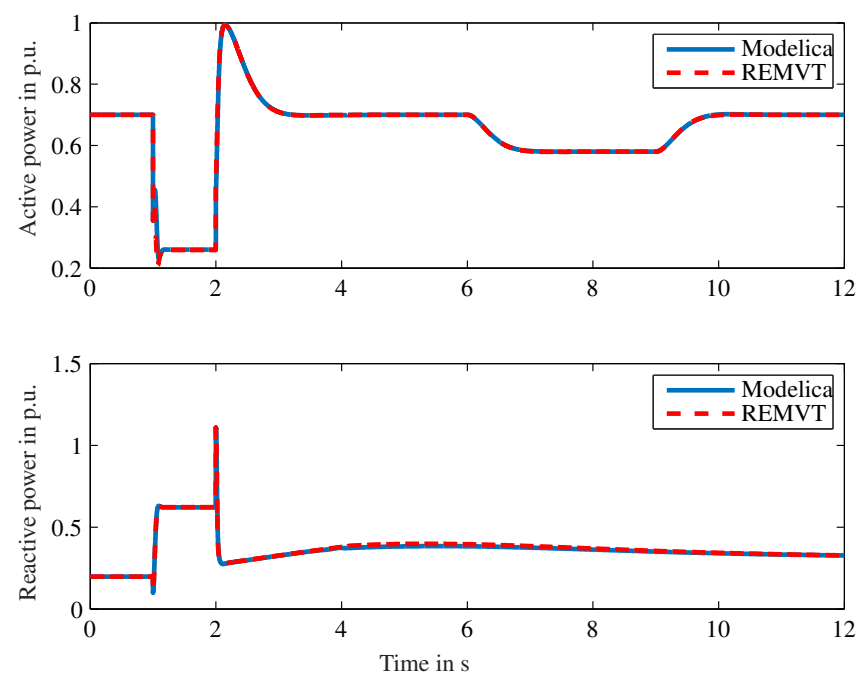

Figure 14. Simulation results $P V$ model: active and reactive power in response to test events with control mode Nr. 4

As also the voltage source interface introduced in (Pourbeik 2018) has been implemented in Modelica, simulation results obtained from OpenModelica have been compared to the reference simulation tools as well.

Currently, the REMVT version does not contain a voltage source network interface. Therefore, if comparing the simulation results of the voltage source interfaced PV model obtained from OpenModelica against the results from REMVT with standard current source interface, the transient results are deviating, but steady state results fit very well. For validation against the reference implementation in PowerFactory, the voltage source interface was available and has therefore been used for validation.

Figure 15 shows exemplarily the validation results from OpenModelica against the reference implementation in PowerFactory for control mode Nr.1, while the models in both simulation environments make use of the voltage source network interface. The results from both simulations environments match perfectly.

\subsection{Validation results - WTG 4A model}

Since the PV and WTG models share large portions of the control structure, the general behaviour of the WTG model without consideration of torsional oscillations (WTG 4B model) and the PV model is similar, therefore only validation results for the WTG 4A model including the drive train representation are presented. Figure 16 shows the validation results for control mode Nr. 4. The results from the Modelica implementation and the validation tool are exactly matching. Also the torsional oscillations in active
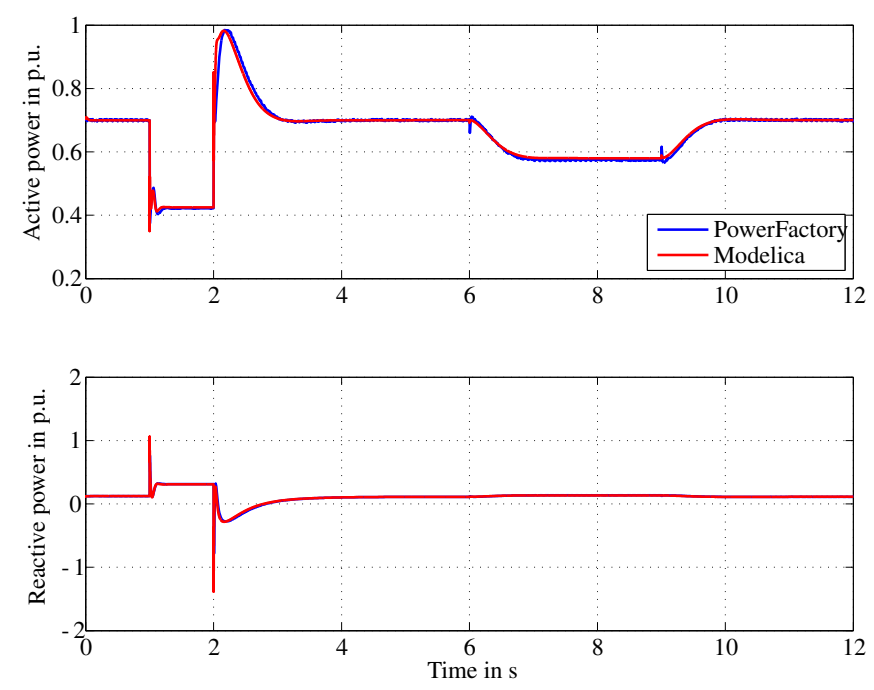

Figure 15. Simulation results PV model: active and reactive power in response to test events with control mode Nr. 1, Voltage source interface

power can be observed.
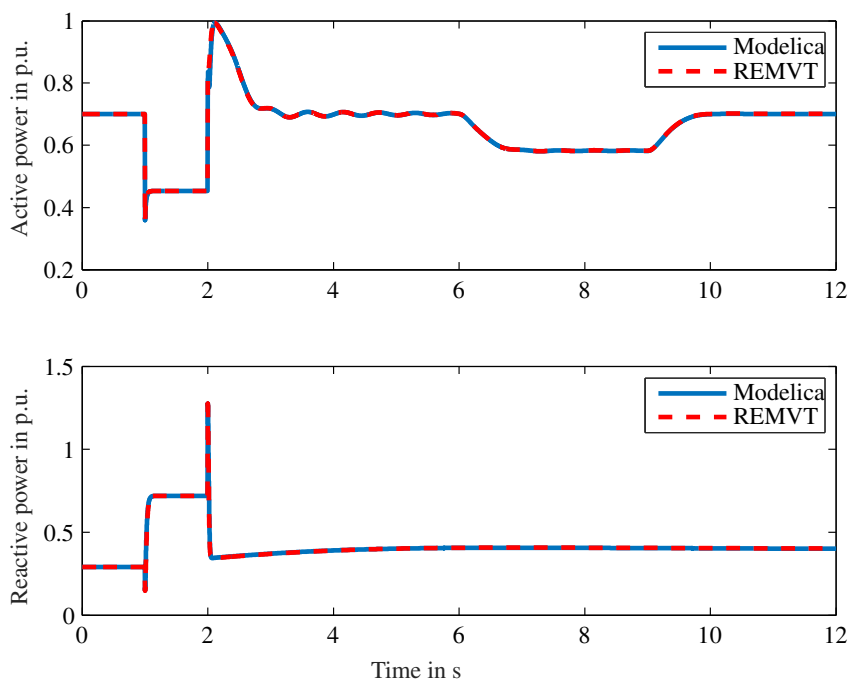

Figure 16. Simulation results WTG 4A model: Active and reactive power response with control mode Nr. 4.

\section{Conclusion}

This paper has presented an open-source implementation of the generic WECC PV and WTG models and their validation against both a validation tool provided by EPRI and an standard library implementation in DIgSILENT/PowerFactory. It demonstrates that the implementation in Modelica of such models is straightforward, easy to understand and to modify thanks to the native properties of the language (declarative and high-level language) and enables to achieve similar accuracy compared to traditional power system simulation tools. It also confirms that Modelica is an appropriate candidate for power system modeling and that its flexibility is a key feature for easy modeling of power-electronics dominated grids. 
In the future, the authors plan to continue their work to both develop open-source Modelica models for standard and advanced power system components and make available standard test cases in order to encourage Modelica use in the power system community. Such an evolution will definitely facilitate technical discussions between all the power system stakeholders, from academics to industrials, and paves the way for a better and more coordinated handling of the numerous challenges arising in power system.

\section{References}

Bartolini, A., F. Casella, and A. Guironnet (2019-02). "Towards Pan-European Power Grid Modelling in Modelica: Design Principles and a Prototype for a Reference Power System Library". In: Proceedings of the 13th International Modelica Conference, Regensburg, Germany, March 4-6, 2019. Linköing University Electronic Press.

Braun, W., F. Casella, and B. Bachmann (2017-05). "Solving large-scale Modelica models: new approaches and experimental results using OpenModelica". In: Proc. 12th International Modelica Conference. Prague, Czech Republic, pp. 557-563.

Casella, F. et al. (2016-10). "Object-Oriented Modelling and Simulation of Large-Scale Electrical Power Systems using Modelica: a First Feasibility Study”. In: Proceedings of the 42nd Annual Conference of the IEEE Industrial Electronics Society IECON 2016. IEEE. Firenze, Italy: IEEE.

Chieh, A., P. Panciatici, and J.Picard (2011-06). "Power system modeling in Modelica for time-domain simulation". In: Proc. PowerTech. IEEE.

Cossart, Q., F. Rosiere, et al. (2020-10). "An Open-Source Implementation of Grid-Forming Converters Using Modelica". In: 2020 IEEE PES Innovative Smart Grid Technologies Europe (ISGT-Europe). IEEE.

Cossart, Q., M. Saugier, and A. Guironnet (2021). An OpenSource Average HVDC Model for Stability Studies. Paper accepted for the 2021 IEEE PowerTech conference.

DIgSILENT/PowerFactory (2019). Template Documentation WECC PV Power Plant Models (PV Models), Revision 1. Tech. rep. DIgSILENT GmbH.

Ellis, E. and P. Pourbeik et.al. (2012). Generic Solar Photovoltaic System Dynamic Simulation Model Specification. Tech. rep. Western Electricity Coordinating Council (WECC) Renewable Energy Modeling Task Force.

Gonzalez-Torres, J.C. et al. (2019). "Power system stability enhancement via VSC-HVDC control using remote signals: application on the Nordic 44-bus test system”. In: 15th IET International Conference on AC and DC Power Transmission (ACDC 2019). Institution of Engineering and Technology.

Guironnet, A., F. Rosière, and G. Bureau (2021). Dynawo: A Suite of Power System Simulation Tools using Modelica and the OpenModelica Compiler. Presentation done at the 2021 OpenModelica virtual Workshop. URL: https://openmodelica. org/events/openmodelica-workshop/openmodelica-program2021.

Guironnet, A., M. Saugier, et al. (2018-10). "Towards an OpenSource Solution using Modelica for Time-Domain Simulation of Power Systems". In: 2018 IEEE PES Innovative Smart Grid Technologies Conference Europe (ISGT-Europe). IEEE.
Henningsson, E., H. Olsson, and L. Vanfretti (2019-02). "DAE Solvers for Large-Scale Hybrid Models". In: Proceedings of the 13th International Modelica Conference, Regensburg, Germany, March 4-6, 2019. Linköing University Electronic Press.

Lammert, G., L. D. Pabon Ospina, and et al. (2017). "Impact of fault ride-through and dynamic reactive power support of photovoltaic systems on short-term voltage stability". In: IEEE Manchester PowerTech, Manchester. IEEE.

Lammert, Gustav, Luis David Pabon Ospina, et al. (2016-07). "Implementation and validation of WECC generic photovoltaic system models in DIgSILENT PowerFactory". In: 2016 IEEE Power and Energy Society General Meeting (PESGM), Boston, MA, USA. IEEE. DOI: 10.1109/PESGM. 2016.7741608.

Lammert, Gustav, Daniel Premm, et al. (2017-03). "Control of Photovoltaic Systems for Enhanced Short-Term Voltage Stability and Recovery". In: IEEE Transactions on Energy Conversion 34, pp. 243-254. DOI: 10.1109/TEC.2018.2875303.

Masoom, A. et al. (2020-12). "Simulation of electromagnetic transients with Modelica, accuracy and performance assessment for transmission line models". In: Electric Power Systems Research 189, p. 106799.

Mirz, M. et al. (2019-07). "DPsim-A dynamic phasor real-time simulator for power systems”. In: SoftwareX 10, p. 100253.

Murad, M.A.A., F. J. Gomez, and L. Vanfretti (2015-06). "Equation-based modeling of FACTS using Modelica". In: 2015 IEEE Eindhoven PowerTech. IEEE.

Nuschke, M. et al. (2019-09). "Power system stability analysis for system-split situations with increasing shares of inverter based generation". In: NEIS conference Hamburg. IEEE.

Pabon Ospina, L. D. and T. Van Cutsem (2020). "Emergency support of transmission voltages by active distribution networks: a non-intrusive scheme". In: IEEE Transactions on Power Systems. DOI: 10.1109/TPWRS.2020.3027949.

Pabon Ospina, L. D. and T.Van Cutsem (2020). "Power factor improvement by active distribution networks during voltage emergency situations". In: Elsevier Electric Power Systems Research Journal 189.

Pabon Ospina, Luis David et al. (2018). "Impact of Plant-Level Voltage Control of Large-Scale Inverter Based Generators on Long-Term Voltage Stability". In: Power Systems Computation Conference (PSCC), Dublin. IEEE. DOI: 10.23919/ PSCC.2018.8442740.

Pourbeik, P. (2018). Proposal for new features for the renewable energy system generic models, 07/23/18, latest revised 3/5/19. Tech. rep.

Qin, Y. et al. (2019-08). "A JModelica.org Library for Power Grid Dynamic Simulation with Wind Turbine Control". In: 2019 IEEE Power \& Energy Society General Meeting (PESGM). IEEE.

Ramasubramanian, D. et al. (2017). "Converter Model for Representing Converter Interfaced Generation in Large Scale Grid Simulations". In: IEEE Transactions on Power Systems 32.

Vanfretti, L. et al. (2013). "Unambiguous power system dynamic modeling and simulation using modelica tools". In: 2013 IEEE Power \& Energy Society General Meeting. IEEE. 